\title{
FISIOLOGÍA MEXICANA EN EL SIGLO XIX: LA ENSEÑANZA
}

\author{
Ana Cecilia Rodríguez de Romo \\ Facultad de Medicina, Universidad Nacional Autónoma de México, México D.F.
}

\section{RESUMEN}

La enseñanza de la medicina en México, permaneció casi la misma los tres siglos de la Colonia. En 1833 el vicepresidente Valentín Gómez Farias reforma la enseñanza y se adopta un programa de estudios médicos muy semejante al francés. Entonces la cátedra de fisiología tomó particular importancia y su evolución a través de los profesores que la impartieron, reflejó claramente el esfuerzo y al mismo tiempo la dificultad para asimilar lo moderno en la fisiología.

\section{SUMMARY}

The Teaching of Medicine in México changed very little during the three centuries of Spanish domination. In 1833, Vice-President Valentín Gómez Parías changed the traditional medical teaching and pushed a program of medical studies very similar to the French. Physiology became very important, its evolution may be followed through the ideas of the professors. This evolution reflects the difficulty to accept new ideas in Physiology and the effort made in order to modernize the Physiology teaching.

\section{INTRODUCCIÓN}

Este análisis se refiere a la historia de la fisiología mexicana en el siglo XIX y que, para fines prácticos, aquí ubicamos entre 1833, año de la creación del Establecimiento de Ciencias Médicas, institución que sistematizó la enseñanza de la medicina, y 1910, inicio de la revolución mexicana. Este capítulo en particular abordará la enseñanza; en uno posterior nos referiremos a la investigación.

Con una profunda influencia francesa, la enseñanza se sistematizó e inició su modernización a partir de 1833. Entonces sucedió un cambio radical en la educación a nivel nacional por razones políticas. En relación a la fisiología, en el México decimonónico no existió una tradición fisiológica, por lo que es difícil marcar una periodización; sin embargo, se puede hablar de un primer periodo o fase de iniciación en el que la enseñanza es más relevante, y de un segundo periodo en el que se sientan las bases de la corriente fisiológica experimental. 
INICIO

El inicio del siglo XIX encuentra en la Nueva España una medicina dogmática que se sigue enseñado de forma casi exclusivamente teórica y apegada a la tradición. Aunque desde el siglo XVII W. Harvey (1578-1657) descubrió la circulación sanguínea utilizando el método experimental ${ }^{1}$, las consecuencias de esta revolución médica y la influencia del método científico se percibieron tarde en México como para reflejarse en la enseñanza al finalizar la Colonia.

Una corriente bien recibida fue el Vitalismo. Los vitalistas X. Bichat (1771-1802) y F. J. Broussais (1772-1788) fueron muy aceptados en México. Esta ideología influyó notablemente el pensamiento fisiológico en México durante la primera mitad del XIX. El historiador de la medicina José Joaquín Izquierdo (1893-1974) pensaba que parte de su buena aceptación se debía a que en nada contradecía las tradiciones y tendencias de la Universidad y la Iglesia².

\section{LA ENSEÑANZA DE LA FISIOLOGÍA, 1833-1917}

De modo muy escueto, se apuntará que el antecedente más remoto de la enseñanza de la fisiología en México sucedió en 1580, cuando la Real y Pontificia Universidad de México estableció la cátedra de Prima Medicina, que también fue el principio de la enseñanza de la medicina ${ }^{3}$.

No es exagerado sugerir que la enseñanza de la medicina no cambió sustancialmente durante los tres siglos de la Colonia. Enfrascada en los tratados del ente y en las abstracciones de la lógica y la metafísica, a la Universidad y al Protomedicato no les interesaban los estudios o reflexiones basados en la experimentación y cualquier novedad era vista con recelo; Hipócrates, Galeno y Avicena seguían dictando la pauta. A finales del siglo XVIII, Carlos III emitió una cédula que entre otros mandaba que el profesor de medicina explicara «el uso de las partes de anatomía», quizá preludio de una cátedra formal de fisiología ${ }^{4}$. Por entonces se inició el movimiento de renovación con un incipiente periodismo científico y el nacimiento del Real Colegio de Cirugía y el Jardín Botánico.

En 1831, se produjo un suceso crucial en la historia medicocientífica mexicana. El Protomedicato fue sustituido por la Facultad Médica del Distrito Federal. En 1833

1 IZQUIERDO, J. J. (1936), Harvey, iniciador del método experimental. Ed. Ciencia, México.

2 IZQUIERDO, J. J.(1936 b), Balance cuatricentenario de la fisiología en México. Ed. Ciencia, México, p. 130.

3 Ibidem, p. 17.

4 Férnandez del Castillo, F., y Castañeda Velasco, H. (1986), Del Palacio de la Inquisición al Palacio de la Medicina. Universidad Nacional Autónoma de México, México, p. 63. 
y durante la primera reforma liberal, el médico Valentín Gómez Farías(1781-1858), entonces vicepresidente del país, reformó la educación y creó el Establecimiento de Ciencias Médicas, que inspirado en el modelo francés, rompió con la tradición en la enseñanza médica. En materia de textos, adoptó los de vanguardia en Francia. Así aparece por vez primera la cátedra de fisiología en el nuevo programa de estudios médicos. La clase se impartía junto con la de higiene 5 . E1 primer profesor fue Manuel Carpio y los textos utilizados las obras de Magendie y Tourtelle. Es significativo que se haya escogido la obra de F. Magendie (1783-1855), pues el destacado fisiólogo francés postulaba que la fisiología requería de la experimentación empírica y de la química y la física, ideas innovadoras y necesarias para la fisiología mexicana.

\section{CUATRO PERIODOS}

La enseñanza pudiera dividirse en cuatro períodos de acuerdo al papel que desempeñaron los profesores: Manuel Carpio (1797-1860) que ya fue mencionado, Ignacio Alvarado (1829-1904), José María Bandera (1832-1910) y Daniel Vergara Lope (1865-1935). Curiosamente, esta división en la enseñanza de la fisiología, coincide con épocas definidas de la historia de México.

En un periodo de iniciación y salvo breves suspensiones, Carpio ocupó la cátedra 27 años, de 1833 hasta 1860, fecha de su muerte. Él caracterizó el primer período de la enseñanza de la fisiología porque sin imprimirle ideas grandiosas, tuvo el mérito de modernizarla. A su respecto, F. Flores (1852-1931) afirma que en el aula era entusiasta y progresista y que fue el primero en hacer demostraciones in vivo ${ }^{6}$. Carpio era admirador de Magendie, aprobaba las teorías de Sydenham pero no estaba de acuerdo con Brown ni con Broussais sobre la «medicina fisiológica».

En esos años de vida independiente, México no había tenido paz, desarrollo económico, concordia social ni estabilidad política; además hubo múltiples epidemias que asolaron a la población. En 1847 sucedió la invasión de Estados Unidos que culminó con la pérdida de más de la mitad del territorio nacional, produciéndose terribles pugnas entre liberales y conservadores. En este panorama, el presidente Benito Juárez (1806-1872) concretó las «Leyes de Reforma»(1859) que limitaban los privilegios de la Iglesia e impulsaban la educación 7 .

El segundo período podría calificarse de «científico» y está representado principalmente por Ignacio Alvarado. Gran admirador de Claudio Bernard (1813-1878) y

\footnotetext{
5 Flores De Asís, F. (1888), Historia de la Medicina en México. Oficina Tipográfica de la Secretaría de Fomento, México, vol. 3, p. 481.

6 Ibidem.

7 Una excelente obra sobre historia mexicana y de fácil acceso es Historia General de México, El Colegio de México, México, (1987), 2 volúmenes. 
fiel seguidor del positivismo, su enseñanza estaba apegada a los cánones de la medicina científica; sin embargo, parece que sus clases eran principalmente teóricas a pesar de ser partidario de la experimentación ${ }^{8}$. Alvarado impartió la cátedra entre 1862 y 1863 y de 1867 a 1876, fecha en que la abandona alegando motivos de salud. Al iniciarse el segundo período de Alvarado en 1867, la fisiología se separó de la higiene, que después se asociaría con meteorología médica9 ${ }^{9}$. Es interesante señalar que en ese mismo año el emperador Maximiliano de Habsburgo (1832-1868) fue fusilado, concluyendo así el Segundo Imperio y dando lugar a lo que se conoce como la República Restaurada con el presidente Juárez otra vez a la cabeza. Sin embargo, una vez más el país estaba destruido por diez años continuos de guerra por la intervención francesa.

José María Bandera fue el catedrático de fisiología en el tercer período que corrió de 1876 a 1909. Izquierdo afirma que entonces hubo una cierta decadencia de la enseñanza porque Bandera no se interesaba en la filosofía científica ni en la fisiología experimental; no organizó las clases prácticas y no se aproximó a las nuevas corrientes externas. Según Izquierdo la enseñanza de la fisiología permaneció en una fase de letargo durante su período docente ${ }^{10}$. Quizá tal opinión es excesiva si se considera el interés de Bandera por la investigación y que se reflejó en sus trabajos: por ejemplo, publicó una casuística de pacientes con enfermedad de Addison que muestra un gran rigor clínico y respaldo anatomopatológico en la mayoría de los casos ${ }^{11}$. Además, desde 1889 , los programas de estudio señalaban una parte teórica y otra experimental y aunque pudiera ser que la idea estuviera en el papel pero no en la realidad ${ }^{12}$, como se enfatizará más adelante, Bandera también era el profesor de prácticas.

Una vez más es interesante mencionar el paralelismo histórico, ya que la época que corre de 1877 a 1910 se conoce como Porfiriato por suceder la dictadura de Porfirio Díaz. El país estaba sediento de paz y aunque entonces se dieron los aspectos negativos de las dictaduras, también hubo calma social que permitió el desarrollo de las ciencias y las artes.

Un último período le correspondería a Daniel Vergara Lope que ejerció la cátedra de 1909 a 1914. De acuerdo con las fuentes, con él se consolidó la enseñanza de la fisiología ya que Vergara Lope fue un científico experimentalista en toda la exten-

8 IZQUIERDO, J. J. (1936 b), pp. 172-177.

9 FloRES DE Asís, F. (1888), pp. 484.

10 IZQUIERDO, J. J. (1951), «Panorama de la fisiología en México hacia el inicio del quinto siglo de vida de su Universidad Nacional Autónoma». Revista de la Sociedad Mexicana de Historia Natural, vol. 12, pp. 46-47.

11 BANDERA, J. M. (1880), «Apuntes para el estudio de la enfermedad de Addison en México», Gaceta Médica de México, pp. 309

12 «La Secretaría de Justicia e Instrucción Pública pide a la Dirección de la Escuela de Medicina un informe sobre el proyecto de reforma del plan de estudio», Archivo Histórico de la Facultad de Medici$n a$, legajo 152, expediente 110, 7 enero 1889. 
sión del término. Fisiólogo teórico y experimental, debe haber representado el ideal del maestro de las disciplinas básicas. Vergara Lope vivió la efervescencia de la Revolución Mexicana que estalló en 1910.

La cátedra de fisiología contó con otros profesores, médicos o investigadores destacados, pero cuyo período docente fue menor y por lo tanto sin repercusiones.

\section{ENSEÑANZA PRÁCTICA DE LA FISIOLOGÍA}

A pesar de todo lo expuesto, quizá en 1833 el cambio en la enseñanza de la medicina, no sólo de la fisiología, no fue todo lo radical que se hubiera deseado. Claro que en las aulas ya no se repetía de forma dogmática las ideas de los antiguos, pero los autores extranjeros eran los únicos que dictaban la pauta, circunstancia que en un principio tuvo ventajas pero que al mismo tiempo estaba lejos de la realidad mexicana. Curiosamente, a mediados del siglo, todavía la parte práctica seguía apoyándose parcialmente en los textos galénicos de tendencia fisiológica ${ }^{13}$.

Las palabras de F. Flores son muy elocuentes al referirse a la enseñanza práctica de la fisiología en el último cuarto del XIX.

\footnotetext{
«El estado actual de la enseñanza de la Fisiología en el país es avanzado, y va al corriente de los progresos que hace en Europa; pero si hemos de ser francos, la cátedra de la Escuela ni está suficientemente dotada, ni se pueden hacer por lo mismo, en ella, las frecuentes vivisecciones y los estudios microscópicos que los continuos adelantos de la ciencia requieren. Es una de las cátedras de nuestra Escuela que necesita urgentemente serias y trascendentales reformas. Y si esto decimos de la cátedra de la capital, ya se comprenderá el estado que debe guardar en las otras Escuelas médicas de la República» ${ }^{14}$.
}

La explicación de Izquierdo es muy realista pues menciona que no había laboratorios de enseñanza porque nadie los reclamaba aunque se vislumbrara su necesidad ${ }^{15}$. Incluso, como lo dice el mismo Flores, no se contaba con una sala adecuada para la clase de fisiología.

Ya se mencionó que en el plan de estudios médicos de 1889, se indica que desde ese año se impartía «fisiología teórica y experimental» ${ }^{16}$; sin embargo hasta 1900 no se crea el primer laboratorio de fisiología en la Escuela de Medicina. El doctor Fernando Altamirano (1848-1907), primer director del Instituto Médico Nacional (18881915), es el encargado de hacer la lista del equipo necesario. Altamirano era además

13 IzQuiERdo, J. J. (1949), Raudón, cirujano poblano de 1810. Ed. Ciencia, México, p. 111.

14 FLORES DE Asís, F. (1888), p. 484.

15 IZQUIERDO, J. J. (1936 b), p. 245

16 «a Secretaría de Justicia...» (1889). 
el jefe del laboratorio de fisiología en la sección tercera del Instituto, laboratorio considerado como el primero de fisiología experimental en el país. El comisionado para desempacar y montar el equipo fue Daniel Vergara Lope, que había adquirido experiencia importante en laboratorios de Moscú, San Petesburgo y París ${ }^{17}$. En 1909, nueve años después, el médico Alfonso Pruneda apuntó que entonces la cátedra de fisiología contaba con el equipo necesario para la parte experimental ${ }^{18}$.

Los temas que se enseñaban en las prácticas, eran los mismos que se impartían en otras escuelas de medicina fuera de México, es decir, fisiología general, las llamadas funciones de la nutrición, de relación, fisiología cardíaca, respiratoria y algunos tópicos del sistema nervioso. El programa de prácticas formulado por Vergara Lope en 1910 , toca aspectos relativos al manejo de animales y equipo elemental de laboratorio, como hacer fístulas, la repetición de experimentos de Claudio Bernard con sustancias tóxicas y experimentos basados en neumografía, esfigmometría, cardiografía y miografía ${ }^{19}$. En 1917, Fernando Ocaranza (1876-1965) propone ante las autoridades de la Escuela de Medicina un programa de prácticas de laboratorio, claramente basado en el método experimental y muchos de los temas de la fisiología digestiva abordados por Bernard. Este programa no fue aprobado ${ }^{20}$. Es interesante resaltar que tanto Vergara Lope como Ocaranza, consideraron formativo para los estudiantes repetir los experimentos del fisiólogo francés.

\section{TEXTOS DE APOYO PARA LA CÁTEDRA DE FISIOLOGÍA EN EL SIGLO XIX}

Es útil hablar un poco acerca de las obras utilizadas para impartir el curso de fisiología, pues dan una idea desde otro punto de vista, del estado de la enseñanza de esta disciplina en México.

Las obras de texto por excelencia, eran los tratados de fisiología de Beclard, Bérard, Duval y Magendie ${ }^{21}$, todos textos franceses. La influencia francesa en la medicina mexicana encuentra aquí un punto más de apoyo. Aunque se utilizaban como libros de consulta los de Müeller ${ }^{22}$ o Tiedemann ${ }^{23}$ que estaban en la biblioteca de la

17 Vergara Lope, D. (1889), «Visita a los laboratorios de fisiología de las Universidades de Moscú, San Petersburgo y Berlín». Anales del Instituto Médico Nacional, vol. 3, p. 175. «Los laboratorios de fisiología en la Sorbona y Bruselas». Anales del Instituto Médico Nacional, vol. 3, p. 361.

18 PRUNEDA, A. (1909), «La enseñanza de la medicina en México». La Escuela de Medicina, p. 114.

19 IZQUIERDO, J. J. (1936 b), p. 153.

20 RODRÍGUEZ DE ROMO, A. C. (1990), «La digestión de las grasas en el programa de fisiología del año 1917: ¿Influencia de C. Bernard en F. Ocaranza?». Revista de la Facultad de Medicina, pp. 697-698.

21 Beclard, J. (1853), Manuel de Physiologie. París. BÉRARD, P. H. (1841-1851), Cours de Physiologie. París. Duval, M. (1887), Cours de Physiologie d'après l'enseignement du Professeur Küss. Paris. Magendie, F. (1836), Précis élémentaire de Physiologie. París, 4 ed., 2 vols.

22 MüEller, J. (1845), Manuel de Physiologie de l'homme. París. 
Escuela y de los que ya había traducción española, no se tomó mucho de la fisiología alemana que también atravesaba su época dorada.

\section{CONCLUSIÓN}

La enseñanza de la fisiología en el siglo XIX mexicano, dependió de modo importante del acontecer histórico, político y social del país. Durante la Colonia, la fisiología y la medicina en general, se enseñaron apegadas a la tradición de los antiguos. En 1833 se dio un cambio dramático, pues el programa de estudios médicos se reformó siguiendo el modelo francés y la cátedra de fisiología adquirió gran importancia. La modernización no fue fácil y el esfuerzo de los catedráticos por adaptarse al cambio es evidente, además de que curiosamente reflejan el momento histórico de la época en que les toca ser profesores de fisiología.

Manuel Carpio, médico moderado, no le imprime originalidad a la fisiología, pero es consciente de su importancia en los estudios médicos y trata de actualizar su enseñanza. Alvarado se volverá radicalmente a la fisiología de laboratorio, Bandera duda entre la fisiología teórica y la aplicada a la clínica y Vergara Lope finalmente representa el fisiólogo de vanguardia que trata de llevar a las aulas lo último de la fisiología científica y la medicina experimental. Los periodos de Bandera y Vergara Lope coinciden con la época de paz y bonanza científica que propicia la dictadura de Porfirio Díaz.

La fisiología se arraigó con fuerza en la mentalidad del joven médico mexicano. Es interesante ver la abundancia de tesis sobre fisiología que los estudiantes hicieron para graduarse a finales del siglo pasado. El «pensamiento fisiológico» que tanto defendió Fernando Ocaranza, fue el preludio del pensamiento patológico, fundamento de una de las épocas más brillantes de la clínica mexicana.

\section{AGRADECIMIENTO}

Se agradece el apoyo otorgado por la Dirección General de Asuntos del Personal Académico, DGAPA, UNAM, través del proyecto IN 402996.

23 Tiedemann, F. (1830-1836), Physiologie de l'homme. Darmstadt. 